\title{
Un domaine privilégié pour l'écologie du sol : le Cabardès
} Jacques Hubschman

\section{Citer ce document / Cite this document :}

Hubschman Jacques. Un domaine privilégié pour l'écologie du sol : le Cabardès. In: Revue géographique des Pyrénées et du Sud-Ouest, tome 43, fascicule 2, 1972. Actes du premier colloque sur la science du paysage. pp. 233-246;

doi : https://doi.org/10.3406/rgpso.1972.3330

https://www.persee.fr/doc/rgpso_0035-3221_1972_num_43_2_3330

Fichier pdf généré le 06/04/2018 


\section{Résumé}

Dans le Cabardès, la diversité des styles de pédogénèse reflète celle des milieux écologiques. Dans le Haut Cabardès occidental, le climat déjà semi-montagnard, la fréquence des vieilles landes, expliquent l'existence, à côté des sols bruns acides modaux, de sols bruns acides dégradés (ocreux) et de sols ocres-podzoliques. Dans certaines cuvettes d'arénisation, on note la présence de sols noirs humifères à profil de type $\mathrm{AC}$ (sols cryptopodzoliques humifères ?). Les sols bruns modaux à mull forestier ne s'observent que sur les versants des gorges, sous taillis de chênes hybrides. Sols bruns acides peu humifères et rankers d'érosion se partagent l'essentiel des bas versants du massif ancien non calcaire et témoignent déjà des influences méditerranéennes. Causses des revers de cuestas et calcaires massifs primaires composent, pour leur part, un domaine méditerranéen édapho-climatique où richesse en calcaire et phénomènes d'érosion, actuels et anciens, commandent la pédogénèse : lithosols caldques, xérorendzines caillouteuses, rendzines rouges, rendzines modales, sols bruns calcaires, s'organisent en mosaïques complexes ou en courtes chaînes de versant. Dans l'ensemble du Cabardès, l'évolution actuelle est celle d'une lente brunification des profils, liée à la progression de la végétation naturelle.

\section{Zusammenfassung}

Im Cabardes deutet die verschiedenartige Pedogenese die Verschiedenheit der ökologischen Milieus an. Im westlichen Haut Cabardès gibt es braune, saure, ausgelaugte (ockerfarbene) Böden und ockerfarbene podsolische Böden, neben braunen, sauren typischen braunen, sauren Böden. Das Vorkommen dieser Böden lasst sich durch das Mittelgebirgsklima und das häufige Antreffen alter Heiden erklären. In gewissen Grusmulden stellt man im Querschnitt das Vorhandensein schwarzer Humusböden vom Typ AC (crypto-podsolische Humusböden) fest. Die typischen braunen Waldmullböden kann man nur im Unterholz hybrider Eichen an den Abhängen der Schluchten feststellen. Braune, saure, wenig humushaltige Böden und Erosionsranker bilden den Hauptanteil der unteren Abhänge des alten, nicht kalkhaltigen Massivs und zeigen schon die Einflüsse des Mittelmeers an. Die Causses auf der Rückseite der Cuestas und primare Kalkmassive bilden ihrerseits ein edapho - klimatisches Mittelmeergebiet, wo reiche Kalkvorkommen sowie derzeitige und alte Erosionserscheinungen die Pedogenese bestimmen : Kalksteinbôden, Schotterxerorendzine, rote Rendzine, typische Rendzine, braune Kalkböden sind in Mosaikform oder in kurzen Catenas an den Abhängen angeordnet. Im ganzen Cabardès stellt man eine derzeitige Entwicklung fest, nämlich die Schichten werden im Querschnitt immer brauner. Das hängt mit dem Fortschreiten der natürlichen Vegetation zusammen. 
REVUE GÉOGRAPHIOUE dES PYRÉNÉES ET DU SUD-OUEST TOME 43, FASC. 2, pp. 233-246, Toulouse, 1972

\title{
Un domaine privilégié pour l'écologie du sol : le Cabardès
}

\author{
par Jacques Hubschman*
}

\begin{abstract}
Autant l'approche analytique, chaque facteur pris un à un, apparaît comme relativement aisée, autant l'appréhension globale de l'ensemble des agents pédogéniques s'avère délicate. Ce problème de "déterminisme combinatoire ", commun il est vrai à la plupart des naturalistes et déjà largement débattu, fournit le thème majeur des recherches de géographie globale sur les milieux "naturels". Pour le pédologue, qui cherche avant tout à définir et à classer des processus, ces études intégrées sont du plus haut intérêt. Elles composent en effet une sorte de canevas général où les principaux facteurs de la pédogénèse, dans leur hiérarchisation comme dans leurs différents niveaux de combinaisons, émergent peu à peu à travers l'ébauche d'une classification des paysages. La mise en évidence de ces unités à la fois physionomiques et dynamiques ne revêt cependant toute son importance que là où facteurs et variables écologiques sont suffisamment diversifiés : climat, lithologie, relief, végétation, mise en valeur. La multiplicité des combinaisons retentit pareillement sur les pédogenèses et autorise l'étude comparative de milieux éco-pédologiques différenciés. A l'est de Toulouse, le versant sud de la Montagne Noire répond parfaitement à ces conditions. Zone de contact entre massif ancien et bassin sédimentaire tertiaire, zone de transition entre climats aquitain à l'ouest et semi-montagnard en altitude et climat méditerranéen de la dépression de Carcassonne, cette région a fait récemment l'objet de deux études de géographie globale conduites à l'échelle de $1 / 20000^{e}$ et de $1 / 25000^{\mathrm{e}}(1)$. En dépit des échelles choisies et de l'orientation
\end{abstract}

* Maître-assistant à l'Université de Toulouse-Le Mirail.

(1) Cf. supra, p. 207-232 et bibliographie in fine. 
spécifique (cartographie des "paysages»), il est possible de dégager de ces recherches et d'une série d'observations connexes, quelques éléments d'une écologie régionale des sols portant sur l'ensemble du Cabardès. Très différencié dans le détail, celui-cj présente néanmoins un certain nombre de caractères communs à la région.

\section{Traits communs à l'ensemble du Cabardès}

\section{La diversité des paysages souligne des oppositions structurelles}

Ces oppositions apparaissent entre nature lithologique d'une part, configuration morphologique d'autre part.

De l'ouest à l'est du Cabardès, le contact entre massif ancien et bassin sédimentaire s'organise suivant un système de cuestas dont les fronts escarpés s'opposent aux plateaux calcaires faiblement inclinés vers le sud des revers. Percées cataclinales aux versants raides et mobiles, vallées sèches remblayées viennent rompre de temps à autre la monotonie de ces paysages de causses. Sur le massif ancien, entaillé de gorges et modelé en pentes modérées, gneiss et granulite, à l'ouest, s'associent aux séries schisteuses primaires dans lesquelles s'intercalent, plus à l'est, calcaires et calcaires dolomitiques primaires. Sols, végétation et modalités de l'exploitation anthropique composent des paysages variés qui reflètent cette diversité.

\section{Un milieu de soulane depuis longtemps marqué par l'homme}

Qu'il ait fait place aux cultures (céréales, fourrage, vigne) ou aux prairies, ou qu'il ait constitué des zones de parcours pour les troupeaux ou une réserve pour l'exploitation du bois, le manteau forestier originel est très largement dégradé : la série du Chêne pubescent au nord-ouest, celles du Chêne vert au sud et à l'est, se présentent sous forme de taillis plus ou moins clairs, de maquis et garrigues, de landes et de friches étroitement associées aux parcelles de culture et constituant une mosaïque complexe et mouvante. Dans ces conditions, la notion de "pédoclimax absolu » actuel perd toute signification.

\section{Un milieu où l'emprise hưmaine s'est relâchée}

La déprise agricole, phénomène très général, ne revêt pas partout la même ampleur. Alors que l'abandon, parfois ancien 
(Haut Cabardès occidental, causses calcaires des revers de cuestas) a été rapide et se poursuit encore de nos jours, les cultures, la vigne surtout, se sont au contraire maintenues et même concentrées dans certains secteurs favorables (dépression périphérique, fond des percées cataclinales, bas versants mollement ondulés du massif ancien à l'est). Dans l'ensemble du Cabardès, la déprise se traduit évidemment par une progression de la végétation naturelle (localement d'origine subspontanée: pinèdes) qui manifeste sous de multiples formes les divers stades de la reconquête. Selon les circonstances, les sols vont porter l'empreinte plus ou moins nette de ce dynamisme.

\section{Des milieux éco-pédologiques typés profondément différenciés dans le détail}

\section{Le domaine semi-montagnard du Haut Cabardès occidental}

Dans le Haut Cabardès occidental (Saissac-St-Denis), la surface polycyclique, taillée dans les gneiss et les schistes du massif ancien, découpée en gorges profondes, présente sur les interfluves une alternance de bossellements et de cuvettes d'arénisation, vestiges de la morphogénèse et de la pédogénèse tertiaire. L'altitude $(500$ à $600 \mathrm{~m}$ ) et la longitude (influence atlantico-aquitaine) déterminent le caractère déjà semi-montagnard du climat: précipitations élevées (moyenne annuelle supérieure à 1 mètre), brouillards fréquents, neige relativement abondante et persistante, températures hivernales basses (moyenne des minimums de janvier inférieure à $1^{\circ} \mathrm{C}$ ), gels fréquents. Ce climat déjà rude est cependant marqué par des étés dont la chaleur et la sécheresse relative sont renforcées par la position de soulane. La série de végétation, dominée par le Chêne pubescent et le Châtaignier, s'exprime en formations dégradées de reconquête : friches, landes, taillis...

Sur les versants en roches acides et compactes (gneiss et schistes) les sols, peu profonds $(30 \mathrm{~cm}$ en moyenne), offrent un faciès jeune proche de celui des rankers (profil A (B) C, parfois A C). Sous taillis, les sols bruns-acides à mull oligotrophe représentent sans doute le stade le plus proche du pédoclimax théorique illustré par une "brunification acide». La percolation des solutions selon la pente, l'acidité d'un milieu par ailleurs vigoureusement aéré et maintenant floculé le complexe argilo-humique, interdisent tout phénomène de "lessivage " généralisé. La série des sols bruns-acides sur gneiss, très acides et de texture grossière (tableau I), s'oppose à celle des sols bruns-acides sur schistes, à $\mathrm{pH}$ plus élevé et plus riches en argile. En fait, deux facteurs écologiques essentiels : dynamique de la végétation et modalités du relief, viennent infléchir l'orientation de la pédogénèse. 
TABleau I - Sol BRUN-ACide « MODAL » SUR GNeiss.

\begin{tabular}{|c|c|c|c|c|c|c|}
\hline \multicolumn{2}{|c|}{$\begin{array}{l}\text { PROFONDEUR } \\
(\mathrm{cm})\end{array}$} & $\begin{array}{c}\% \\
\text { ARgILE }\end{array}$ & $\mathrm{pH}$ & $\begin{array}{l}\text { MATIÈRE } \\
\% \text { ORGA- } \\
\text { NIQUE }\end{array}$ & $\mathrm{C} / \mathrm{N}$ & $\begin{array}{c}\text { FER } \\
\text { «LIBRE } \\
\%\end{array}$ \\
\hline $\mathrm{Al}$ & $5-15$ & 6,0 & 4,9 & 3,2 & 13 & 0,63 \\
\hline (B) & $15-25$ & 52 & 4,7 & 0,8 & 10 & 0,59 \\
\hline
\end{tabular}

(*) Fer « libre» selon méthode Deb (hyposulfite).

a) Dynamique de la végétation : sols bruns-acides "dégradés"et sols ocres-podzoliques des bossellements gneissiques. Labours et landes se partagent ici l'essentiel de la surface. Cultures et labours ont homogénéisé le sol et accéléré la combustion des matières organiques: les taux de carbone sont inférieurs à $1 \%$ et des éléments grossiers de roche et de (B) cambique affleurent en surface.

La lande jeune, caractérisée par la présence de ronces et de Prunus spinosa en mélange avec Ulex europaeus et Sarothamnus scoparius, tend déjà à reconstituer un profil "naturel » où sous une mince litière, l'horizon $\mathrm{Al}$ se différencie à nouveau de l'horizon cambique. Seule, une maigre frange superficielle, acidifiée ( $\mathrm{pH}$ inférieur à 4,5) par rapport au sol brun-acide "modal ", témoigne de l'influence du tapis végétal.

La lande «mûre" polyspécifique (Ulex europaeus, Erica scoparia, Calluna vulgaris, Pteridium aquilinum), parfois arborée, est associée à des sols qui, en première analyse, se rattachent au type brun-acide. En fait, la litière plus abondante, la structure moins ferme de Al (mull moder) le $\mathrm{pH}$ nettement plus acide (de l'ordre de 4) et l'horizon cambique parfois légèrement ocreux, semblent devoir rapprocher ces formations des sols bruns-cryptopodzoliques à moder $(4,6)$. Faute de données suffisamment précises, nous ne pouvons que les qualifier de sols bruns-acides " dégradés ".

Sous les vieilles landes monospécifiques (Sarothamnus scoparius surtout et Ulex europaeus), la dynamique du fer et de l'aluminium, commandée par la nature du métabolisme biochimique du xéromoder (A0 net, A1 particulaire) et déterminée par l'ambiance ionique ( $\mathrm{pH}$ très bas), se traduit dans les profils, essentiellement par l'apparition d'une bande rouille en profondeur, riche en fer et en matière organique (tableau II), typique du sol ocre-podzolique (horizon spodique discontinu). 
Tableau II - Sol ocre-Podzolique SUR GNeISS.

\begin{tabular}{|c|c|c|c|c|c|c|}
\hline \multicolumn{2}{|c|}{$\begin{array}{l}\text { PROFONDEUR } \\
(\mathrm{cm})\end{array}$} & \multirow{2}{*}{$\begin{array}{c}\% \\
\text { ARGILE } \\
8,1\end{array}$} & \multirow{2}{*}{$\begin{array}{c}\mathrm{pH} \\
3,9\end{array}$} & \multirow{2}{*}{$\begin{array}{c}\text { MATIÈRE } \\
\% \text { ORGA- } \\
\text { NIQUE } \\
6,2\end{array}$} & \multirow{2}{*}{$\frac{\mathrm{C} / \mathrm{N}}{21}$} & \multirow{2}{*}{$\begin{array}{c}\begin{array}{c}\text { FER } \\
\text { " LIBRE } \\
\%\end{array} \\
0,36\end{array}$} \\
\hline $\mathrm{Al}$ & $5-15$ & & & & & \\
\hline B & $25-30$ & 6,9 & 4,2 & 22 & 19,2 & 0,7 \\
\hline
\end{tabular}

De même que certaines de ces vieilles landes, où les arbres s'installent très difficilement, peuvent être considérées comme paraclimaciques, de même le sol ocre-podzolique peut apparaître, dans cette optique, comme un para-pédoclimax de dégradation : il est en effet douteux que, dans ces conditions d'aération, de drainage et surtout de roche-mère (gneiss compact), la série évolutive ainsi esquissée se poursuive par des stades podzoliques plus élaborés à $\mathbf{A} 2$ cendreux ou à $\mathrm{Bh}$ nets, par exemple.

Sur schiste, on n'observe jamais, semble-t-il, d'évolution de ce type : l'argile libérée en abondance au cours de l'altération de ce matériau joue ici son rôle de stabilisateur d'humus et freine considérablement les processus.

b) Les cuvettes d'arénisation : des sols cryptopodzoliques humifères? Les interfluves gneissiques sont marqués par l'alternance de bossellements amples et de cuvettes d'arénisation très humides l'hiver, desséchées pendant les mois d'été. Celles-ci sont généralement occupées par de vieilles prairies mal entretenues, envahies par les espèces acidiphiles de la lande (Ulex, Pteridium). Elles portent un sol rankériforme (fig. 1) épais de 30 à $50 \mathrm{~cm}$, de couleur noire, très riche en matière organique (tableau III). Les profils, acides, sont relativement homogènes : la couche supérieure $(A 0: 0-10 \mathrm{~cm})$ présente néanmoins une structure moins fine que celle de l'horizon profond où apparaissent des éléments d'arène. L'ensemble repose soit sur le gneiss en place soit, plus fréquemment, sur un mince manteau arénacé parsemé de débris gneissiques.

Tableau III - Sol Noir humifère de cuvette.

\begin{tabular}{|c|c|c|c|c|c|}
\hline $\begin{array}{c}\text { PROFONDEUR } \\
(\mathrm{cm})\end{array}$ & $\begin{array}{l}\text { Couleur } \\
\text { Munsell }\end{array}$ & $\begin{array}{c}\text { ARGILE } \\
\%\end{array}$ & $\mathrm{pH}$ & $\begin{array}{l}\text { MATIÈRE } \\
\% \text { ORGA- } \\
\text { NIQUE }\end{array}$ & $\mathrm{C} / \mathrm{N}$ \\
\hline Ao $\quad 0-10$ & 10 YR $2 / 1$ & 8,2 & 4,6 & 28,8 & - \\
\hline (AIB) ? 20-30 & 10 YR $2 / 2$ & 9,4 & 4,9 & 8,7 & 15,2 \\
\hline
\end{tabular}


L'analogie avec certains types de sols décrits comme rankers pseudo-alpins ou sols cryptopodzoliques-humifères $(2,3)$ est frappante. Rappelons que ces sols sont caractérisés par une matière organique abondante et très bien humifiée et des teneurs en Fe et Al libres relativement importantes dans tout le profil. Une fois encore, les données beaucoup trop fragmentaires dont nous disposons ne nous permettent pas de nous prononcer sans réserves sur cette parenté. On pourrait cependant s'étonner d'observer, dans les conditions écologiques du Haut-Cabardès occidental, un type de sol qui a été décrit soit à altitude plus élevée (pelouse alpine ou pseudo-alpine, altitude supérieure à $1000 \mathrm{~m}$ : Vosges), soit en région atlantique (ranker atlantique) le long des côtes de Galice et du Portugal (7). En fait, il semble que l'ambiance

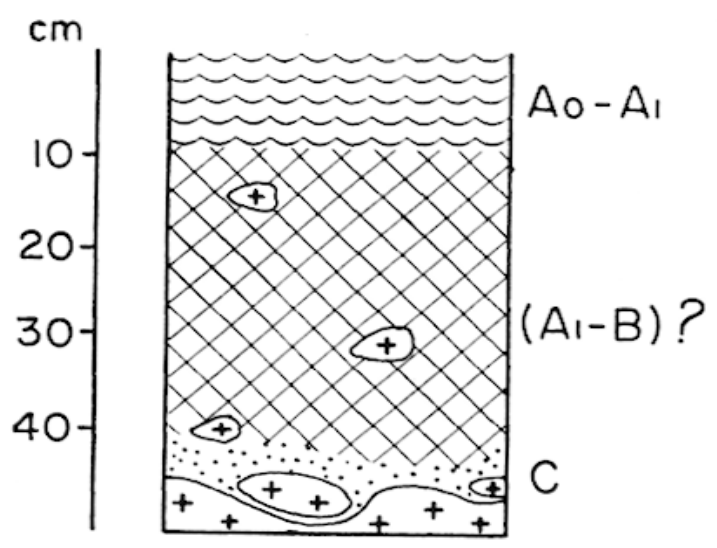

FIG. 1

Sol humifère des cuvettes d'arénisation. Profil schématique.

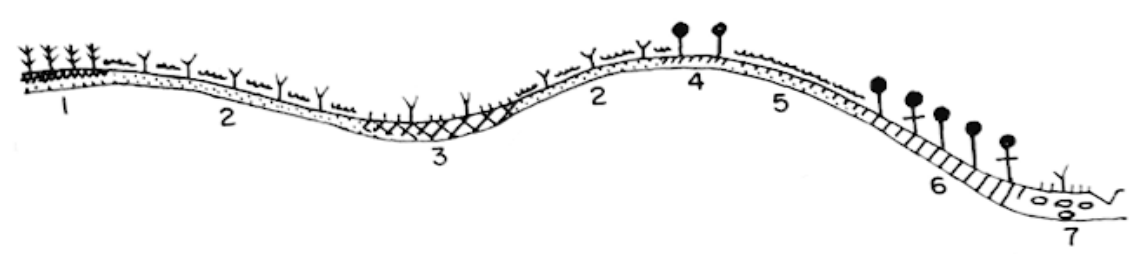

FIG. 2

Juxtaposition, séquence et chaînes intraséquentielles de sols sur gneiss en Haut Cabardès occidental.

1. Sol ocre-podzolique (vieille lande à Sarothamne). - 2. Sol brun-acide "dégradé " (friche-lande et lande mûre). - 3. Sol humifère (cryptopodzolique ?) des cuvettes d'arénisation (prairie-lande). - 4. Sol brun-acide rankériforme (taillis clair de Chêne pédonculé, à ronces). - Sol brun-acide perturbé (labours, friches récentes). - 6. Ranker à mull et sol brun modal (taillis mixte à Chêne pédonculé, Chêne hybride). - 7. Sol brun à pseudogley (prairie-lande hygrophile). 
écologique qui regne en Haut-Cabardès soit a priori parfaitement compatible avec une genèse de ce type. En effet, les hivers rudes, longs et neigeux permettent l'accumulation d'importantes quantités de matière organique et la libération momentanée de composés solubles agressifs vis-à-vis du fer et de l'aluminium qui se trouvent bientôt complexés. Les étés, relativement chauds et arides, favorisent la minéralisation et la précipitation in situ des composés organo-métalliques dans des cuvettes d'arénisation par ailleurs brutalepnent asséchées. Enfin, ces cuvettes d'arénisation constituent des pièges pour les solutions organiques qui percolent sur les pentes au cours de l'hiver. Dans cette optique, les sols humifères de cuvette représenteraient ainsi le terme aval de courtes chaînes de versant (fig. 2) formées, à l'amont, par les sols bruns-acides de pente. Ceux-ci, depuis longtemps dépourvus de leur couverture forestière dense, légèrement dégradés, sont le siège d'une libération ménagée de complexes organiques solubles à fer et alumine, qui viennent s'accumuler dans les cuvettes. Dans l'hypothèse où le type cryptopodzolique-humifère de ces sols noirs viendrait à être confirmé (teneur en $\mathrm{Fe}$ et $\mathrm{Al}$, taux d'humification), on peut se demander si sa genèse ne résulte pas de la combinaison de deux systèmes écologiques différents : un système écologique montagnard, marqué par des hivers humides, froids et neigeux; un système méridional (déterminé ici par l'influence méditerranéenne) à été déjà chaud et sec. Les sols humifères des cuvettes d'arénisation représenteraient alors un intermédiaire entre ranker pseudo-alpin et ranker atlantique.

c) Versants des gorges et replats schisteux : des chaines de sols "classiques". Sur les versants boisés des gorges (taillis exploités de Chênes pubescents et Chênes hybrides - entre pubescents et pédonculés -) les sols épais $(50 \mathrm{~cm})$, développés sur colluvium de pente, présentent une couleur foncée et une structure grumeleuse qui les rapprochent soit des sols bruns modaux à mull forestier (B structural sur schistes) soit des rankers à mull actif (profil A C sur gneiss). Dans les fonds de vallées, l'hydromorphie provoque l'apparition de faciès à pseudogley (fig. 2).

Sur les replats des pentes schisteuses, occupés par la prairie ou la lande arborée, la pédogénèse a pu s'exercer de façon plus " régulière " (manteau colluvial argileux et approfondissement "in situ ") : dans ce matériel homogène et relativement argileux (20\% en moyenne) les sols sont légèrement lessivés (sols brunslessivés) et offrent un $B$ textural déjà net.

Ces types de courtes chaînes "classiques" se retrouvent, avec diverses nuances, sur tout le massif ancien acide du Cabardès, dans des situations écologiques variées. 


\section{Influences méditerranéennes dans la partie méridionale du massif ancien non calcaire}

Granulite et gneiss (à l'ouest) et séries schisteuses se partagent l'essentiel de la surface. L'altitude plus faible (inférieure à $400-500 \mathrm{~m}$ ), la proximité de la dépression de Carcassonne, expliquent les caractères d'un climat aux hivers moins humides et sans neige, aux étés plus chauds et plus secs que dans le HautCabardès occidental. Ce domaine est celui de la série du Chêne vert acidiphile. Ici, les "landes " n'offrent ni le même aspect ni la même composition floristique que dans le Haut-Cabardès : mêlées aux Ericacées et au Sarothamne, les espèces late-méditerranéennes (Cistus salviaefolius, Cistus monspeliensis, Spartium junceum, Lavandula stoechas) deviennent de plus en plus nombreuses de l'ouest à l'est, faisant transition avec les maquis dégradés à Chêne vert clairsemé.

Ces conditions écologiques marquées par une sécheresse certaine, se traduisent dans la pédogenèse. L'érosion par ruissellement plus active (nombreux rankers de pente et lithosols), les sols moins épais et plus caillouteux (surtout à l'est du Cabardès), la faiblesse des taux de matière organique (moins de 2,5\%) due à une combustion accélérée de l'humus, les $\mathrm{pH}$ plus élevés (environ 5), sont autant d'indices d'un style de pédogénèse déjà méditerranéen. Ceci apparaît d'autant plus clairement sur les versants de gneiss et granulite de l'ouest : les sols des cuvettes d'arénisation présentent un type général brun-acide peu humifère analogue à celui des sols de pente, à la différence qu'ils sont développés sur une arène claire et caillouteuse plus profonde.

\section{Causses des revers de cuestas et calcaires massifs primaires}

Sur un substratum sec et à fort pouvoir thermo-réflecteur, la série du Chêne vert calcicole s'exprime en une mosaïque de faciès de dégradation et de progression : taillis clairs, buxaies, garrigues ouvertes et garrigues fermées à Chêne kermès, pelouses sèches à Graminées et à frutescents, pinèdes subspontanées (Pin d'Alep, Pin pignon).

Nulle part mieux que dans ces milieux où la pédogénèse est très lente (calcaires durs), l'impact des défrichements, du pâturage ou de la mise en culture, responsables d'un ruissellement généralisé et encore actif, n'est plus sensible : les sols, peu profonds et souvent caillouteux, forment un manteau discontinu. Sur les revers de cuestas, des pavages d'éléments calcaires gélifractés alternent avec des plages de roches totalement décapées et des xérorendzines et rendzines pauvres en calcaire fin. Sur 
Tableau IV - Sols calcimagnésiques du Cabardès (analyse sommaire).

\begin{tabular}{|c|c|c|c|c|c|c|c|c|c|}
\hline & $\begin{array}{l}\text { PROFONDEUR } \\
(\mathrm{cm})\end{array}$ & Couleur Munsell & $\begin{array}{c}\text { ELÉ- } \\
\text { MENTS } \\
\text { GROS > } \\
2 \mathrm{~mm}\end{array}$ & $\begin{array}{c}\text { ARGILE } \\
\% \\
\text { TERRE } \\
\text { FINE }\end{array}$ & $\mathrm{pH}$ EAU & $\underset{\text { TOTAL }}{\mathrm{Ca} \mathrm{CO}_{3}}$ & $\underset{\text { FIN }}{\mathrm{Ca} \mathrm{CO}_{3}}$ & $\begin{array}{l}\text { MaTIÈRE } \\
\text { \% ORGA- } \\
\text { NIQUE }\end{array}$ & $\mathrm{C} / \mathrm{N}$ \\
\hline 1 & & 2,5 YR $4 / 4$ & 50 & 31,5 & 7,9 & 9,2 & 1,1 & 0,9 & 8,1 \\
\hline 2 & & 2,5 YR $2 / 4$ & 30 & 29,7 & 7,6 & 11,6 & 0,9 & 2,0 & 10 \\
\hline 3 & & 2,5 YR $4 / 6$ & 13,5 & 46,1 & 7,6 & 2,2 & traces & 0,6 & 6,4 \\
\hline 4 & & 10 YR $3 / 2$ & 27 & 27,9 & 8,0 & 12,7 & 4,2 & 3,8 & 9,8 \\
\hline 5 & $0-12$ & 7,5 YR $3 / 2$ & 18 & 33,4 & 6,9 & traces & 0 & 3,2 & 13,6 \\
\hline \multirow{3}{*}{6} & $15-20$ & 25 YR $4 / 4$ & 35 & 30,0 & 7,9 & 7,4 & 1,2 & 1,2 & 9,9 \\
\hline & $0-20$ & $5 \quad$ YR $4 / 3$ & 14 & 41,1 & 8,1 & 5,1 & 0,9 & 1,9 & 10,1 \\
\hline & $20-60$ & $5 \quad$ YR $4 / 4$ & 9,5 & 42,8 & 8,0 & 4,9 & 1,2 & 09 & 7,3 \\
\hline
\end{tabular}

1) Xérorendzine caillouteuse sur calcaire nummulitique (revers de cuesta) : pelouse sèche à frutescents (Lavandula vera, Dorycnium suffruticosum).

2) Rendzine pauvre en calcaire fin sur calcaire nummulitique : taillis de Chêne vert à tapis herbacé à Brachypodium ramosum.

3) Rendzine pauvre en calcaire fin sur calcaire massif primaire : pelouse sèche. 4) Rendzine modale sur colluvium de bas de pente (percées cataclinales) : taillis de Chêne vert à Erica scoparia
et Spartium junceum.

5) Xérorendzine brunifiée sur calcaire nummulitique : Pins d'Alep, Chênes verts. 6) Sol brun-calcaire sur colluvium de vallée sèche (revers de cuesta) : pelouse sèche à Brachypodium ramosum
et Festuca ovina. 
les calcaires primaires, le sol est piégé dans les lapiès et les fissures.

La nature calcaire du substrat impose à la pédogénèse son cadre spécifique : les sols sont en général nettement carbonatés (en moyenne 5 à $10 \%$ de $\mathrm{Ca} \mathrm{CO} 3$ total de la terre fine), parfois magnésiques (sur calcaire dolomitique du Géorgien supérieur de Sallèles). La réaction, neutre ou alcaline, reflète un complexe saturé par le calcium ou le magnésium.

Le matériel matriciel meuble est argileux et rubéfié : les sols peuvent être assimilés dans l'ensemble à des terra-rossa résiduelles légèrement brunifiées, relativement pauvres en calcaire fin (moins de $1 \%$ en moyenne), où l'abondance des fragments rocheux assure un renouvellement continu de la carbonatation. La rubéfaction, évidemment héritée, ne présente à nos yeux aucune signification écologique actuelle ou sub-actuelle : nombre de calcaires compacts ou cristallins libèrent spontanément par dissolution un reliquat d'argile rouge imprégnée d'oxydes de fer deshydratés, sans qu'il soit pour cela nécessaire de faire intervenir des processus actuels ou anciens de rubéfaction bioclimatique $(5,8)$ : le pourcentage de fer "libre " de xérorendzines rouges du Cabardès varie de 1 à 3 . En revanche, la "rougeur " de ces formations témoigne surtout de la faiblesse de la brunification, consécutive à un rajeunissement des sols par ruissellement et corrélative d'une reconquête végétale qui s'amorce à peine.

La multitude de faciès offerts par ces sols calcimagnésiques, outre qu'elle exprime une certaine diversité pétrographique (calcaires cristallins primaires, calcaires tertiaires plus tendres), résulte principalement de la combinaison entre morphogenèse ancienne (vallées sèches, percées cataclinales) et actuelles (dynamique des versants) et caractères du tapis végétal. Certains aspects analytiques en sont présentés dans le tableau IV.

Les rendzines sur calcaires massif sont plus argileuses et surtout moins riches en carbonates que celles des revers de cuestas : la fragmentation du calcaire tertiaire est beaucoup plus poussée (tableau IV : 1, 2, 3). Là où la végétation a subsisté partiellement ou a progressé, les taux de matière organique, bas sous pelouse, s'élèvent sensiblement (tableau IV : 2,5 ). D'autre part, le développement des pinèdes, dont les litières sont riches en composés acides hydrosolubles, infléchit la pédogénèse vers une certaine brunification des rendzines : acidification superficielle et progressive du mull calcique à $C / N$ inférieur à 10 en mull calcique à $\mathrm{C} / \mathrm{N}$ plus élevé (tableau IV : 5 ). Ies rendzines "modales", riches en humus et à structure en grumeaux bien exprimés, ne se présentent qu'en bas de versant (essentiellement sur les pentes des percées cataclinales), sous taillis hygrophile à Ericacées ou sous fruticée. Développées à la faveur d'affleure- 
ments marneux riches en calcaire fin, elles constituent l'aboutissement très classique de courtes chaînes de sols (fig. 3 ) qui s'anastamosent vers l'aval avec la séric des sols bruns-calcaires sur marnes des fonds de percées. Dans les vallées sèches des plateaux calcaires, les sols bruns-calcaires se développent sur un matériel de remblai provenant du décapage des xérorendzines de plateau (fig 3). Moins caillouteux que celles-ci, parfois encore cultivés, ils ne présentent aucun signe d'évolution notable ni vers les sols bruns-calciques $\mathrm{ni}$, a fortiori, vers les sols bruns-lessivés (tableau IV : 6).
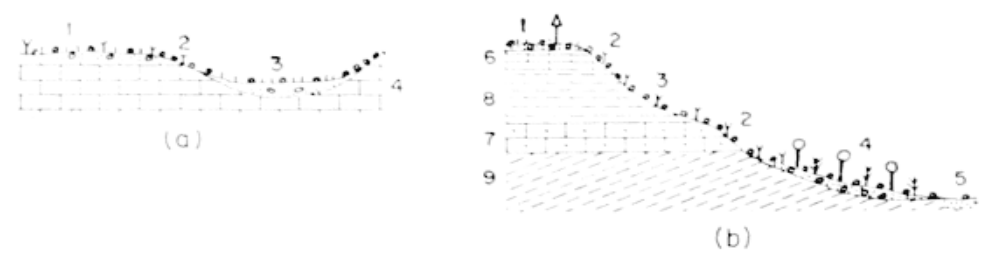

FIG. 3

Courtes chaines "classiques" de versant sur revers de cuestas calcaires.

(a) Vallée sèche : 1. Xérorendzine pauvre en calcaire fin (pelouse sèche a frutescents). - 2. Lithosol calcique (frutescents). - 3. Sol brun-calcaire (pelouse sèche). - 4. Calcaire en dalle.

(b) Versant de percée cataclinale : 1, Xérorendzine pauvre en calcaire fin (pelouse sèche à Pin noir d'Autriche). - 2 . Lithosol calcique. 3. Régosol calcique. - 4. Rendzine "modale" (taillis hygrophile à Chêne vert). - 5. Sol brun-calcaire sur marnes (vignes). - 6-7. Calcaires en dalle. - 8. Argile. - 9. Marnes.

\section{Sols bruts d'érosion des fronts de cuestas}

Ourlant le contact entre massif ancien et bassin sédimentaire, les fronts de cuestas (souvent dédoublés à l'ouest) sont taillés dans les marnes et argiles sparnaciennes et yprésiennes. La forte pente de versants généralement exposés au nord, la faible résistance des roches, l'existence de suintements au contact des plateaux calcaires, déterminent un milieu très particulier où le ruissellement diffus et concentré (ravines) entrave la pédogénèse. L'emprise de la végétation revêt une multitude d'aspects : formation à Schoenus nigricans, Brachypodium pinnatum; Thym, Callune, Chêne kermès à l'est ; stades buissonnants à Spartier et Ericacées, taillis de Chêne vert à Pin sylvestre ou Pin noir d'Autriche à l'ouest. Les sols sont pour l'essentiel des sols bruts d'érosion, proches des régosols (ou des lithosols là où apparaît sur 
le front une corniche thanétienne). Localement, sous une maigre litière de taillis ou de pinède, se développe un mince horizon A1 (1 à $3 \mathrm{~cm}$ d'épaisseur en moyenne). En fait, le seul processus notable actuel est l'hydromorphie (suintements) qui provoque la marmorisation ménagée des marnes brutes (parfois mêlées d'arènes au bas des pentes). Sur la mince bande marneuse qui s'allonge d'ouest en est au pied du front avancé, les sols bruns-calcaires, mal drainés, présentent eux aussi fréquemment des faciès à pseudogley et parfois à gley (ouest du Cabardès).

\section{Un complexe écologique en mutation lente : progression de la végétation naturelle et évolution des sols}

A l'exception de certains secteurs particuliers (concentration des vignes sur les bas versants schisteux du Cabardès oriental), le recul de l'emprise humaine a pour corollaire la reconquête de l'espace par la végétation naturelle. Ce dynamisme ( contrôlé ", il est vrai, par l'homme : coupes, reboisements, exploitation «touristique »...) se manifeste nécessairement dans l'évolution des sols : enrichissement en produits organiques, restauration d'un pédoclimat frais, ralentissement des processus morphogéniques sur les versants...

Sur le massif ancien acide, rankers et sols peu épais ou peu humifères se transforment en sols bruns-acides "modaux ». On peut néanmoins s'interroger sur le sort des sols "dégradés ». Dans l'hypothèse d'une reconquête par le taillis ou la forêt, le renforcement de l'activité biologique (passage d'un mull-moder à un mull acide) doit probablement conduire à une disparition progressive des sols bruns-acides dégradés. En revanche, les sols ocres-podzoliques, nous l'avons vu, correspondent peut-être à un "para-pédoclimax" local associé aux vieilles landes monospécifiques : il est parfaitement possible que, du fait de la concurrence vitale et de l'extrême acidité d'un sol chimiquement très pauvre (tableau II), l'arbre ne puisse s'y installer. Quant aux sols humifères des cuvettes d'arénisation, nous avons estimé que la cryptopodzolisation, proposée avec les réserves d'usage, aurait été induite par la dégradation, largement favorisée par le climat neigeux et à hiver froid, des sols bruns-acides rankériformes des pentes. En bonne logique, le rétablissement d'un profil "modal " stable sur ces pentes doit mettre un terme à l'enrichissement en composés organo-minéraux des sols de cuvette : ceux-ci deviendront alors "fossiles" ou inactifs. Leurs caractères singuliers n'en disparaîtront pas pour autant.

Sur les plateaux calcaires et les calcaires primaires, où la pédogénèse est très lente, sur les fronts de cuestas stabilisés par la 
végétation, une seule évolution perceptible : la brunification superficielle. Ceci est particulièrement sensible dans les pinèdes à progression rapide. Au-dessous de la litière se développe un horizon Al1 frais, à réaction neutre, colonisé par les Annélidés, où le cycle de la matière organique est moins rapide que sous pelouse sèche ou garrigue clairsemée. On retrouve un schéma identique sur les marnes et argiles des fronts de cuestas et des versants de percées cataclinales, colonisés par le taillis hygrophile à Chêne vert ou la pinède. A long terme, la plupart des sols sur calcaire évoluent très classiquement vers la rendzine brunifiée, tandis que les sols bruns-calcaires cemmencent à peine à se décarbonater en surface (tableau IV : 5).

Cette esquisse éco-pédologique du Cabardès fait ressortir les principales combinaisons écologiques qui régissent la pédogénèse : dans la pratique, celles-ci se manifestent par une certaine hiérarchie spatiale des facteurs.

Dans le Haut-Cabardès occidental, le caractère semi-montagnard du climat autorise déjà des phénomènes locaux de podzolisation ménagée.

Rajeunissement des sols et combustion accélérée de la matière organique attestent l'influence méditerranéenne sensible dans tout le bas massif ancien acide. La nature calcaire du substrat, au sud et à l'est, induit une large série de combinaisons à dominante édaphique, à sols calcimagnésiques peu évolués. Sur les fortes pentes marneuses des fronts de cuestas, la pédogenèse est bloquée.

Dans l'ensemble du Cabardès, la progression de la végétation naturelle tend, dans une certaine mesure, à homogénéiser les pédogénèses à travers une brunification lente mais généralisée des profils.

\section{BIBLIOGRAPHIE}

1. G. Cantegril, D. Marty, S. Vié : Etude de géographie globale en Cabardès occidental. Mémoire de maîtrise géographique, Toulouse, 1969. F. LAHÉRA, B. Llenes : Les paysages du Cabardès occidental, idem, ibidem, 1969.

2. T. Carballas, Ph. Dichauloi:R et F. Jacolix : Evolution de la matière organique des Rankers. Bull. E.N.S.A. Nancy, 1967, IX, fasc. 1.

3. R. Carbenier : Remarques sur un type de sol encore peu étudié : le Ranker cryptopodzolique de l'étage subalpin du Massif hercynien français. C. R. Ac. Sc., 1963, 4, 977-979.

4. C.P.C.S. : Commission de Pédologie et de Cartographie des sols. Grignon, 1967.

5. D. Dımas : Contribution à l'étude des terres rouges méditerranéennes. Thèse Doct. $3^{e}$ cycle, Fac. Sc. Toulouse, 1969. 
6. Ph. Dichalfolk et B. Solchitr : Note sur un problème de classification. Podzolisation chimique et différenciation du profil. Pédologie (Gand), 1965, 15, $143-158$.

7. H. Frantz : Drei Klimabedingte Ranker-Subtypen Europas. 6e Congrès int. Sc. du Sol, Paris, 1956, V, 22, vol. E, p. 135-141.

8. J. Hubschman : Limons gris et limons rouges du Quaternaire récent marocain : " provinces pétrologiques " et essai d'interprétation par l'érosion différenticlle. Zeistschrift fiir Geomorphologie, 1971, fasc. 15, t. 3, p. 261-263.

Réscimé - Dans le Cabardès, la diversité des styles de pédogénèse reflète celle des milieux écologiques. Dans le Haut Cabardès occidental. le climat déjà semi-montagnard, la fréquence des vieilles landes, expliquent l'existence, à còté des sols bruns acides modaux, de sols bruns acides dégradés (ocreux) et de sols ocres-podzoliques. Dans certaines cuvettes d'arénisation, on note la présence de sols noirs humifères à profil de type AC (sols cryptopodzoliques humifères?). Les sols bruns modaux à mull forestier ne s'observent que sur les versants des gorges, sous taillis de chênes hybrides. Sols bruns acides peu humifères et rankers d'érosion se partagent l'essentiel des bas versants du massif ancien non calcaire et témoignent déjà des influences méditerranéennes. Causses des revers de cuestas et calcaires massifs primaires composent, pour leur part, un domaine méditerranéen édapho-climatique où richesse en calcaire et phénomènes d'ćrosion, actuels et anciens, commandent la pédogénèse : lithosols calciques, xérorendzines caillouteuses, rendzines rouges, rendzines modales, sols bruns calcaires, s'organisent en mosaïques complexes ou en courtes chaînes de versant. Dans l'ensemble du Cabardès, l'évolution actuelle est celle d'une lente brunification des profils, liée à la progression de la végétation naturelle.

Zusammenfassung. - Im Cabardes deutet die verschiedenartige Pedogenese die Verschiedenheit der ökologischen Milieus an. Im westlichen Haut Cabardès gibt es braune, saure, ausgelaugte (ockerfarbene) Böden und ockerfarbene podsolische Böden, neben braunen, sauren typischen braunen, sauren Böden. Das Vorkommen dieser Böden lässt sich durch das Mittelgebirgsklima und das häufige Antreffen alter Heiden erklären. In gewissen Grusmulden stellt man im Querschnitt das Vorhandensein schwarzer Humus. böden vom Typ AC (crypto-podsolische Humusböden) fest. Die typischen braunen Waldmullböden kann man nur im Unterholz hybrider Eichen an den Abhängen der Schluchten feststellen. Braune, saure, wenig humushaltige Böden und Erosionsranker bilden den Hauptanteil der unteren Abhänge des alten, nicht kalkhaltigen Massivs und zeigen schon die Einflüsse des Mittelmeers an. Die Causses auf der Rückseite der Cuestas und primäre Kalkmassive bilden ihrerseits ein edapho - klimatisches Mittelmeergebiet, wo reiche Kalkvorkommen sowie derzeitige und alte Erosionserscheinungen die Pedogenese bestimmen : Kalksteinböden, Schotterxerorendzine, rote Rendzine, typische Rendzine, braune Kalkböden sind in Mosaikform oder in kurzen Catenas an den Abhängen angeordnet. Im ganzen Cabardès stellt man eine derzeitige Entwicklung fest, nämlich die Schichten werden im Querschnitt immer brauner. Das hängt mit dem Fortschreiten der natürlichen Vegetation zusammen. 